\title{
Satisfacción de la Calidad Educativa en Educación Superior
}

\section{Satisfaction with Upper Education Quality}

Jayson Andrey Bernate ${ }^{1}$, Alexander Guataquira Romero ${ }^{2}$, Erika Natalia Romero Melo ${ }^{3}$, Paula Carina Reyes Escobar ${ }^{3}$

INFORMACIÓN DEL

\section{ARTÍCULO}

Fecha de recepción: 11 de Mayo de 2020.

Fecha de aceptación: 15 de Octubre de 2020

Doctor en Gerencia y Política Educativa, Universidad de Baja California de México. Docente- Investigador, Corporación Universitaria Minuto de Dios-UNIMINUTO-Colombia.

E-mail: : jbernate1@uniminuto.edu.co Código ORCID:

https://orcid.org/0000-0001-5119-8916

${ }^{2}$ Magíster en Enseñanza de las Ciencias Exactas y Naturales, Universidad Nacional de Colombia. DocenteInvestigador de la Secretaria de Educación SED-BOGOTA- Colombia. E-mail:

alexanderguataquirome@gmail.com

Código ORCID:

https://orcid.org/0000-0001-8208-425X

3 Candidata a Licenciada en Educación Física, Corporación Universitaria Minuto de Dios-UNIMINUTO. Miembro de la Facultad de Educación UNIMINUTO-Colombia. E-mail:

emeloromero@uniminuto.edu.co

Código ORCID:

https://orcid.org/0000-0002-1666-9564

4 Candidata a Licenciada en Educación Física, Corporación Universitaria Minuto de Dios-UNIMINUTO. Miembro de la Facultad deEducaciónUNIMINUTO-Colombia E-mail:

preyesescob@uniminuto.edu.co

Código ORCID:

https://orcid.org/0000-0002-0502-8876

CITACIÓN: Bernate, J.A., Guataquira Romero, A., Romero Melo, E.N., \& Reyes Escobar, P.C. (2020). Satisfacción de la Calidad Educativa en Educación Superior. Podium, 38, 37-50 doi:10.31095/podium.2020.38.3

\section{ENLACE DOI:}

http://dx.doi.org/10.31095/podium.202 0.38 .3

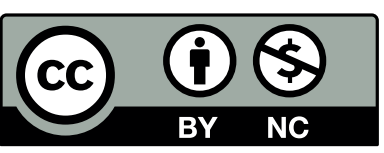

\section{Resumen}

El presente artículo tiene como objetivo analizar el grado de satisfacción que tienen los estudiantes universitarios en un programa de educación superior. Para ello, se realizó la encuesta SEUE elaborada por Gento-Palacios y Vivas-García (2003), compuesta por 93 ítems y 10 variables, la cual fue aplicada a 200 estudiantes de séptimo y octavo semestre. La metodología utilizada fue cuantitativa y el diseño descriptivo. Los principales hallazgos reflejan que los grupos II, IV, VII y X, arrojan puntuaciones satisfactorias respecto a la media, mientras que los grupos I y VI, presentan puntuaciones poco satisfactorias respecto a la media y los grupos III y IX presentan puntuaciones muy satisfactorias respecto a la media. Se concluye que es imperante medir los procesos educativos para mejorar las condiciones de los programas.

\section{Palabras Clave:}

Satisfacción, educación superior, enseñanza, estudiantes, calidad, universidad.

\section{Clasificación JEL: I20.}

\begin{abstract}
This article's aims is to analyze university students' degree of satisfaction in an upper education program. SEUE survey, which was prepared by Gento-Palacios y Vivas-Garcia (2003), was given to 200 seventh and eighth semester students; this survey consisted of 93 items and 10 variables. The study used a quantitative methodology and a descriptive design. The main findings reflect that groups II, IV, VII and X, show satisfactory scores with respect to the median, while groups I and VI, show scores that are not very satisfactory with respect to the median and groups III and IX show very satisfactory scores with respect to the median. The conclusion is that it is imperative to measure educational processes in order to improve program conditions.
\end{abstract}

\section{Keywords:}

Satisfaction, upper education, teaching, students, quality, university.

JEL Classification: I20. 


\section{Introducción}

La educación superior en Latinoamérica muestra continuas transformaciones, Colombia es claro ejemplo de ello puesto que a través del tiempo los sistemas de percepción de calidad en programas universitarios cuentan con diferentes herramientas tecnológicas que miden periódicamente los procesos curriculares, pedagógicos, logísticos, etc. Esto fue creado desde los estándares de autoevaluación permanente del Ministerio de Educación Nacional (MEN), con el fin de garantizar las mejoras continuas de los programas en educación superior. Uno de los aspectos de mayor importancia al medir la calidad organizacional, es evaluar la satisfacción de los usuarios, siendo los estudiantes actores principales de las instituciones universitarias, quienes tienen mayor relevancia para evaluar la calidad de los servicios educativos.

En la misma línea Mejías y Martínez (2009) proponen una metodología de diez pasos para la medición de la satisfacción estudiantil en instituciones de educación superior (IES). Esta metodología con su enfoque sistémico y sistemático, presenta carácter dinámico, lo que permite su adaptación a otros entornos. Además, constituye un aporte para que las Instituciones de Educación Superior puedan mejorar continuamente con la intención de lograr una mejor Calidad Educativa, puesto que el estudiante sería un cliente satisfecho. También Surdez, Sandoval, y Lamoyi (2018) aportaron al modelo de satisfacción universitaria identificando las oportunidades de mejora para incrementar el grado de satisfacción estudiantil en referencia a infraestructura y retroalimentación positiva del trato respetuoso que reciben los estudiantes de sus tutores, y a las oportunidades que les brinda la institución para su desarrollo personal. Asimismo, especifican diferencias de satisfacción estadísticamente significativas entre grupos de edad, campus al que pertenece el estudiante, programas de estudio, y estado civil.

Por otro lado, es claro que las condiciones de calidad que rigen un programa de educación superior están sucedidas de múltiples factores, en esta investigación se abordarán los siguientes: Condiciones básicas de infraestructura, servicios de la institución, condiciones de seguridad, consideración a su situación económica, seguridad emocional por el trato afectuoso, sentido de pertenencia a la institución y al grupo de clase, proceso de enseñanza y aprendizaje, logros personales y reconocimiento al éxito personal. Igualmente, se puede señalar que esta investigación está encaminada a evaluar la satisfacción que tienen los estudiantes de educación superior respecto a su programa académico.

En efecto, la calidad educativa de un estudiante universitario está ligeramente ligada al bienestar que este pueda obtener en su ámbito de formación educativa (Aguilera García, 2010). De acuerdo a lo anteriormente planteado el objetivo de esta investigación es analizar el grado de satisfacción que tienen los estudiantes universitarios en un programa de educación superior. 


\section{Revisión de literatura}

El concepto de calidad se basa en que los usuarios sean servidos al máximo grado posible, significando que los servicios o productos satisfagan sus requerimientos y necesidades. El usuario juzga si la calidad de los bienes y servicios es aceptable y satisface sus necesidades y es él quien debe fungir como centro de cualquier organización que busque la excelencia (Clemenza, Ferrer y Pelekais, 2005). En lo que respecta al contexto latinoamericano, Orozco Cruz, Olaya Toro, y Villate Duarte (2009) destacan el carácter polisémico del concepto de calidad y los retos que plantea a la educación superior, en su devenir cotidiano, asumir una perspectiva de la calidad ligada tanto a las demandas de los contextos educativos reales como a las expectativas de los diferentes actores sociales que cohabitan por la institucionalidad educativa y con la concreción de una perspectiva de derechos en las prácticas escolares, que potencie la subjetividad política, en particular de los estudiantes universitarios. De igual manera, Alcántar Enríquez, Maldonado-Radillo, y Arcos Vega (2012) sostienen que la gestión de la calidad en la educación superior promueve cambios positivos al interior de la universidad en cuatro componentes básicos: dirección y liderazgo, desarrollo de procesos académicos, desempeño de los equipos de trabajo y comportamiento de los actores individuales. En este sentido, Villarruel Fuentes (2010) menciona que los Sistemas de Gestión de Calidad se esgrimen dentro de las IES Latinoamericanas como una alternativa de trabajo académico, cuyos objetivos son sistematizar los métodos y procedimientos administrativos, fomentar la cultura de servicio y capacitar al personal, en busca de la satisfacción del cliente (estudiante).

Álvarez Botello, Chaparro Salinas, y Reyes Pérez (2015) afirman que la calidad es elemento clave para que una organización sea competitiva. Sin embargo, al ser la calidad una característica tan dinámica y específica para cada sector, es necesario contar con las herramientas adecuadas para evaluarla. Por ende, las universidades necesitan un sistema de evaluación y acreditación que les permita participar activamente en el proceso de globalización académica

Además, al momento de aplicar los modelos de calidad educativa se pueden enfocar varios inconvenientes o problemáticas respecto al entorno del aprendizaje en los estudiantes como en la enseñanza de los docentes (Bianchetti, 2017). A pesar de las mejoras a nivel gubernamental en contratación $\mathrm{y}$ capacitación docente, aún se encuentran lejos de lo ideal los presupuestos asignados para la educación, como es el caso de Colombia, donde anualmente estos son mínimos para apuntarle a una revolución de la calidad en educación. Por consiguiente, se ha podido evidenciar en algunas instituciones públicas y privadas el deterioro de infraestructura de los centros educativos, generando inconformidades continuas en docentes y estudiantes, conduciendo a que se realicen paros cívicos regularmente, 
trayendo consigo otras problemáticas a nivel social y cultural. Ratificando lo anterior, Camacho y Delgado Noguera (2002) afirman que "la calidad en la educación debe de ser producida desde periodos tempranos" (p. 11), es decir que la empiecen a aplicar desde los colegios, ofreciendo desde edades iniciales protocolos pre establecidos para optimizar los procesos de educación en los estudiantes, y de igual manera reforzando los aprendizajes previos, teniendo en cuenta que estos aprendizajes no sólo irían articulados a nivel cognitivo, sino también a nivel socio afectivo y psicológico. En este caso y para poder trabajar los aspectos anteriores (cognitivo, socio afectivo y psicológico), se debe primero detectar en los alumnos sus fortalezas y debilidades. En Colombia se deben articular dichos procesos en los docentes, ya que a veces se da prioridad solamente a lo académico y no a lo emocional, es decir que esta es una dimensión de estudio que debería ser trabajada por los académicos de forma continua en todos los procesos de enseñanza y aprendizaje y sus niveles

También se puede inferir que el grado de satisfacción que consigue el estudiante en el desarrollo de su formación académica se ven reflejados en los resultados alcanzados durante el proceso de su formación profesional, y en el avance de sus metas educativas a corto, mediano y largo plazo. Puesto que, animar $\mathrm{o}$ asistir en los procesos formativos de los educandos, forman parte de mecanismos y reacciones que van ligados a la parte psicológica de cada estudiante (Fernández-Abascal y Jiménez
Sánchez, 2010). Debido a que el estudiante, cuando se siente plenamente apoyado, logra con certeza y seguridad resultados satisfactorios hacia las capacidades que pueden desarrollar y experimentar. Además, es de resaltar que los espacios de infraestructura que la institución les ofrece a los estudiantes para desarrollar sus aprendizajes son otros de los factores que inciden en la satisfacción del educando, al igual que las ayudas financieras que estas les proporcionan y el acompañamiento constante de profesionales expertos en materia de sus conocimientos. Gran parte de estos aspectos van ligados al grado de satisfacción que pueda obtener el estudiante en la institución y de la comodidad de esta (Cadena-Badilla, Mejías-Acosta, Vega-Robles, y Vásquez Quiroga, 2016).

Igualmente, se puede dar referencia a las metodologías que se utilizan en las aulas de clase, y cómo estas influyen en los estudiantes, de manera positiva o negativa. Puesto que ello, es un factor determinante en la percepción del estudiantado, ya que un estudiante de educación superior percibe de manera dinámica cuando un docente tiene un alto o bajo nivel de apropiación de las temáticas y conceptos a trabajar en la clase (Guzmán, 2011). Es decir, que si el educador presenta falencias en el aula de clase frente al dominio de temáticas o aplicación de estrategias esto podría llevar a la insatisfacción en los procesos de formación del educando. Lo anterior se complementa con el argumento de Vega Cantor (2014) quien explica que al pasar los años el significado de calidad se 
ha ido cambiando hacia un enfoque empresarial, usando términos como mejoramiento y control de calidad; es decir, que los estudiantes pasarán a ser objetos o productos, lo cual su único objetivo será mejorar los resultados. Mientras que Dos Santos (2016) alude que, aunque la calidad es un constructo multidimensional, la estructura física juega un papel fundamental, porque las instalaciones materiales tienen un impacto en la satisfacción de los clientes. Además, Bernate, Fonseca, y Betancourt (2019) manifiestan que la calidad educativa tiende a tornarse como un acto complejo debido a que este entra a un campo de transformación con base en unas necesidades sociales, lo que también se vislumbra en Contreras, Caballero, Palacio, y Pérez (2008) pues exponen que la educación es un derecho fundamental y que este va ligado a la permanencia y la calidad de la educación al interior de una sociedad; ambas son importantes en la educación, ya que la primera hace relación a la garantía de la educación como derecho primordial y la segunda es más un criterio y se relaciona con la acción educativa.

\section{Metodología}

El método de la investigación es cuantitativo de diseño descriptivo, ya que como lo sostienen Hernández-Sampieri, Fernández-Collado, \& Baptista Lucio (2010) este es un método que intenta recopilar información cuantificable para ser utilizada en el análisis estadístico de la muestra de una población. Es una herramienta popular de investigación de mercados que permite recopilar y describir la naturaleza del segmento demográfico.

La muestra de esta investigación fue de tipo aleatorio constituida por 200 estudiantes de $7^{\circ}$ y $8^{\circ}$ semestre del programa de Licenciatura en Educación Física, Recreación y Deportes de la Corporación Universitaria Minuto de Dios, sede principal Bogotá-Colombia; de los cuales 44 son de género femenino y 156 de género masculino, con edades entre los 19 a los 38 años y con estratos sociodemográficos de 1, 2, 3 y 4; como es el caso de Colombia en donde 1 es el más bajo y 4 es el más alto, distribuidos de la siguiente manera: $60 \%$ es de estrato 2 , $35 \%$ de estrato $3,4 \%$ es de estrato $1 \mathrm{y}$ finalmente el $1 \%$ es de estrato 4 . Además, el $2 \%$ se encuentra en edades de 19 a 20 años, el $80 \%$ está en el rango de edad de los 20 a 26 años, $16 \%$ está entre los 26 y los 30 años y finalmente el $2 \%$ de la población se encuentra entre los 30 y 38 años de edad.

El instrumento que se utilizó fue el SEUE, encuesta para conocer la satisfacción de los estudiantes universitarios con su educación, elaborada por Gento-Palacios y Vivas- García (2003). La encuesta consta de 93 ítems y se divide en 10 grupos: grupo I. Condiciones básicas de infraestructura (13 ítems), grupo II. Servicios de institución (13 ítems), grupo III. Condiciones de seguridad (6 ítems), grupo IV. Consideración a su situación económica ( 7 ítems), grupo V. Seguridad emocional por el trato afectuoso (8 ítems), grupo VI. Sentido de pertenencia a la institución y al grupo de clase por la aceptación (10 ítems), grupo VII. Proceso 
de enseñanza y aprendizaje (19 ítems), grupo VIII. Logros personales (6 ítems), grupo IX. Reconocimiento del éxito personal (5 ítems) y grupo $\mathrm{X}$. Autorrealización (6 ítems). El formato de respuesta fue por medio de una escala tipo Likert con 5 opciones de respuesta: Bastante satisfecho (5), muy satisfecho (4), satisfecho (3), poco satisfecho (2), y Tabla 1 .

Fiabilidad del test

\begin{tabular}{lcc} 
Fiabilidad del test & & \\
\hline \multicolumn{1}{c}{ Variables } & $\begin{array}{c}\text { Alpha de } \\
\text { Cronbach }\end{array}$ & $\begin{array}{c}\text { Número de } \\
\text { elementos }\end{array}$ \\
\hline Grupo I. Condiciones básicas de infraestructura &, 755 & 13 \\
Grupo II. Servicios de la institución &, 655 & 13 \\
Grupo III. Condiciones de seguridad &, 580 & 6 \\
Grupo IV. Consideración a su situación económica &, 843 & 7 \\
Grupo V. Seguridad emocional por el trato afectuoso &, 984 & 8 \\
Grupo VI. Sentido de pertenencia a la institución y al &, 849 & 10 \\
grupo de clase & & 19 \\
Grupo VII. Proceso de enseñanza y aprendizaje &, 655 & 6 \\
Grupo VIII. Logros personales &, 873 & 5 \\
Grupo IX. Reconocimiento al éxito personal &, 815 & 6 \\
Grupo X. Auto relación &, 832 & 93 \\
\hline Escala Total &, 784 & \\
\hline
\end{tabular}

Fuente: Elaboración propia.

Tabla 2

Variables e ítems del instrumento

\begin{tabular}{|c|c|}
\hline Grupo I. Condiciones básicas de infraestructura & Grupo II. Servicios de la Institución \\
\hline 1. Limpieza de las instalaciones. & 14. Servicio bibliotecario (atención al usuario). \\
\hline 2. Ventilación de las aulas. & 15. Dotación de la biblioteca. \\
\hline 3. Aislamiento del ruido. & 16. Oficina de registro y control estudiantil. \\
\hline 4. Comodidad del mobiliario. & 17. Proceso de admisión e inscripción. \\
\hline 5. Espacios para la enseñanza. & 18. Acceso a computadoras para traba. Académicos. \\
\hline 6. Espacios para el estudio. & 19. Acceso a internet y correo electrónico. \\
\hline 7. Instalaciones sanitarias. & 20. servicio de reproducción y fotocopiado. \\
\hline 8. Disponibilidad del agua potable. & 21. Higiene de los alimentos en el comedor. \\
\hline 9. Espacios para el descanso. & 22. Cantidad de alimentos ofrecidos. \\
\hline 10. Espacios para la recreación. & 23. Calidad de alimentación. \\
\hline 11. Instalaciones de la biblioteca. & 24. El apoyo que brinda el centro de estudiantes. \\
\hline 12. Instalaciones del comedor. & 25. Servicio médico - odontológico. \\
\hline 13. Instalaciones deportivas. & 26. Transporte. \\
\hline
\end{tabular}

totalmente insatisfecho (1). En la Tabla 1 se detalla la fiabilidad del test demostrando que la información suministrada es confiable para la investigación.

El instrumento se divide en 10 variables, las cuales a su vez se subdividen en ítems que están agrupadas tal como se muestra en la Tabla 2. 
Tabla 2.

Variables e ítems del instrumento

Grupo III. Condiciones de seguridad

27. Del edificio (salidas de emergencia y extintor)

28. Del mobiliario (sin aristas peligrosas, materiales rompibles o inflamables).

29. Del transporte (del vehículo de la ruta).

30. De las zonas de esparcimiento.

31. De las instalaciones deportivas.

32. De los alrededores de la institución.

Grupo V. Seguridad emocional por el trato afectuoso
Grupo IV. Consideración a su situación económica

33. Las actividades académicas.

34. Las actividades extra cátedras.

35. El servicio de comedor.

36. El servicio de transporte.

37. Los servicios médico - asistenciales.

38. La asignación de becas.

39. La adquisición de materiales de estudio.

Grupo VI. Sentido de pertenencia a la institución y al grupo de clase
40. Sus profesores.

41. Sus compañeros de grupo.

43. Las autoridades de la Universidad.

44. El personal administrativo.

45. El personal de la biblioteca.

46. El personal del comedor.

47. El personal del servicio médico.
42. Los otros alumnos de la Universidad.

48. Las autoridades.

49. Los jefes de departamentos.

50. los profesores.

51. El personal administrativo.

52. El personal de servicio.

53. Los compañeros de clase.

54. El contexto social por ser estudiante de la insta.

55. Consideración y respetos a sus ideas o propuestas (aula).

56. Representación en las diversas instancias.

57. Participación en las políticas y proyectos institucionales.

Grupo VII. Proceso de enseñanza y aprendizaje

58. El plan de estudios de la carrera.

59. El contenido de las asignaturas.

60. El régimen de estudios (anual/semestral).

61. Procesos de admisión e inscripción.

62. Los materiales impresos y audiovisuales disponibles.

63. Dominio de los contenidos y actualizaciones de los profesores.

64. El nivel de exigencia.

65. Los sistemas de evaluación.

66. La orientación y apoyo de los profesores en las horas de consultas.

67. La metodología de la enseñanza.

68. La comunicación con los profesores en el aula.

69. La formación práctica.

70. La vinculación con el contexto nacional y regional.

71. La vinculación con los futuros centros de trabajo.

72. La asistencia a clase de los profesores.

73. El acceso a las diversas manifestaciones culturales (teatro, cine y conciertos).

74. Los recursos extra cátedras para optimizar su formación.

75. Incorporación de las nuevas tecnologías a las enseñanza.

76. El servicio de asistencia y asesoramiento psicológico.

\section{Grupo VIII. Logros personales}

77. Con los resultados o calificaciones obtenidas.

78. Con los conocimientos adquiridos.

79. Con el dominio de las técnicas de estudio.

80. Con la formación ciudadana.

81. Con la habilidades y destrezas para el trabajo docente desarrolladas

82. Con el desarrollo integral de su persona.

Grupo IX. Reconocimiento del éxito personal

83. La institución y sus autoridades.

84. Los profesores.

85. Los alumnos de su grupo.

86. Los demás alumnos de la universidad.

87. El contexto social.

Grupo X. Auto - relación

88. Poder hacer lo que verdaderamente quiere y está en la capacidad de hacer.

89. Saber que cada día va adquiriendo mayor conocimiento y destrezas.

90. La libertad de que goza en la institución.

91. La autonomía de que goza en la realización de sus trabajos.

92. El desarrollo de su creatividad.

93. Su futuro profesional.

Fuente: Gento-Palacios y Vivas-García (2003) 
Para realizar la recolección de los datos, se informó a los participantes sobre la investigación. Se pidió la colaboración de los docentes y de los estudiantes. Se entregó un formato dirigido a los estudiantes solicitando su consentimiento para vincularse al estudio. El día de la aplicación de la encuesta se explicó el objetivo y las variables que tenía la realización de la misma. Los estudiantes tuvieron un tiempo de 40 minutos para contestar la encuesta de manera auto determinada.

El análisis estadístico se realizó por medio del programa SPSS 23.0. Se hizo un análisis de la fiabilidad del instrumento, se calcularon los estadísticos descriptivos de las variables y dimensiones del estudio, teniendo en cuenta la media aritmética y desviación típica.

\section{Resultados}

Al realizar los análisis e interpretación de datos que arroja la aplicación del instrumento (ver Tabla 3), se observa la media aritmética más baja en el grupo I: Condiciones básicas de infraestructura, por ende la población estudiantil está poco satisfecha en esta variable, ya que se presenta una desviación típica baja, lo cual indica que los datos están dispersos o distribuidos cerca a la media, equivalente a 0,71. Al reconocer esta dificultad de planta física es importante citar a Clemenza et al. (2005), quienes recomiendan optimizar los bienes y servicios de infraestructura en educación superior.

Por otra parte, en el grupo II: Servicios de la institución, se observa satisfecha la población estudiantil en esta variable, lo que resulta de gran importancia según el aporte de Camacho y Delgado Noguera (2002) en donde resaltan la necesidad de acompañar el bienestar psicológico de los estudiantes.

Tabla 3 .

Estadísticos descriptivos de las variables de estudio

\begin{tabular}{lcc}
\hline \multicolumn{1}{c}{ Variables } & $\begin{array}{c}\text { Media } \\
\text { Aritmética }\end{array}$ & $\begin{array}{c}\text { Desviación } \\
\text { Típica }\end{array}$ \\
\hline Grupo I. Condiciones básicas de infraestructura & 2,15 & 0,71 \\
Grupo II. Servicios de la institución & 3,87 & 0,73 \\
Grupo III. Condiciones de seguridad & 4,23 & 0,65 \\
Grupo IV. Consideración a su situación económica & 3,76 & 0,76 \\
Grupo V. Seguridad emocional por el trato afectuoso & 4,21 & 0,56 \\
Grupo VI. Sentido de pertenencia a la institución y al & 2,21 & 0,58 \\
grupo de clase & & \\
Grupo VII. Proceso de enseñanza y aprendizaje & 3,45 & 0,65 \\
Grupo VIII. Logros personales & 3,87 & 0,64 \\
Grupo IX. Reconocimiento al éxito personal & 4,20 & 0,63 \\
Grupo X. Auto relación & 3,98 & 0,76 \\
\hline
\end{tabular}

Fuente: Elaboración propia. 
Al reflejarse una desviación típica baja, se indica que los datos están dispersos o distribuidos cerca a la media, equivalente a 0,73 .

En lo que respecta al grupo III: Condiciones de seguridad, se obtuvo la media aritmética más alta, con lo que se ratifica el alto nivel de satisfacción en el que se encuentra la población estudiantil, ya que se presenta una desviación típica baja, indicando que los datos están dispersos o distribuidos cerca a la media, equivalente a $0,65 \mathrm{y}$, coincidiendo con lo contemplado por Orozco Cruz et al. (2009) quienes explicitan que la calidad en la educación no se reduce a un área específica sino que contempla multiplicidad de factores que potencian el proceso de enseñanza aprendizaje.

En el grupo IV: Consideración a su situación económica, se presenta una desviación típica baja, lo cual indica que los datos están dispersos o distribuidos cerca a la media, equivalente a 0,76 , reflejándose satisfacción en la población estudiantil, lo que se puede contrastar con los argumentos de Alcántar Enríquez et al. (2012) quienes afirman que incluir el tema de calidad ha sido decisivo para los grandes avances frente al desarrollo de los procesos en la educación.

En el grupo V: Seguridad emocional por el trato afectuoso, se obtuvo la segunda media aritmética más alta, con esto se evidencia muy satisfecha la población estudiantil, ya que se presenta una desviación típica baja, lo cual indica que los datos están dispersos o distribuidos cerca a la media, equivalente a 0,56. Al respecto Álvarez et al. (2015) enfatizan en la necesidad de revisar los entornos de los estudiantes, ya que estos inciden de manera directa en el desempeño académico y por supuesto en los niveles de calidad alcanzados por las instituciones.

En el grupo VI: Sentido de pertenencia a la institución y al grupo de clase. Se obtuvo la segunda media aritmética más baja, con esto se evidencia poco satisfecha la población estudiantil, ya que se presenta una desviación típica baja, lo cual indica que los datos están dispersos o distribuidos cerca a la media, equivalente a 0,58. Lo que según Vega Cantor (2014) puede deberse a que los estudiantes no deben percibirse como objetos sino como seres activos de su realidad y su proceso de formación.

En el grupo VII: Proceso de enseñanza y aprendizaje, se evidencia satisfecha la población estudiantil, ya que se presenta una desviación típica baja, lo cual indica que los datos están dispersos o distribuidos cerca a la media, equivalente a 0,65 . Nótese entonces que el argumento de Villarruel Fuentes (2010) sobre la capacitación de los docentes permite el dominio de los temas y la riqueza de estrategias para acompañar el aprendizaje, lo que se torna fundamental para lograr la satisfacción del cliente, en este caso los estudiantes.

En el grupo VIII: Logros personales, la población estudiantil se nota satisfecha, ya que se presenta una desviación típica baja, lo cual indica que los datos están dispersos o distribuidos 
cerca a la media, equivalente a 0,64. Es así como Fernández-Abascal y Jiménez Sánchez (2010) afirman que apoyar al estudiante en sus intereses y necesidades será de gran importancia para que logre sus metas personales y profesionales.

En el grupo IX: Reconocimiento al éxito personal, se obtuvo la tercera media aritmética más alta, con esto se percibe muy satisfecha la población estudiantil, ya que se presenta una desviación típica baja, lo cual indica que los datos están dispersos o distribuidos cerca a la media, equivalente a 0,63 . Aquí cobra fuerza lo expresado por Bernate et al. (2019) en cuanto a situar la educación como un acto complejo y de transformación que impacta positivamente al individuo y a la sociedad en la que se encuentra inmerso. Finalmente en el grupo X: Auto relación, se evidencia satisfecha la población estudiantil, ya que se presenta una desviación típica baja, indicando que los datos están dispersos o distribuidos cerca a la media, equivalente a 0,76. En concordancia Contreras et al. (2008) afirman que es necesario reconocer la educación como un derecho, lo que posibilita proceso de empoderamiento $\mathrm{y}$ cambio social.

Los resultados de la investigación se ubican en su mayoría en la escala de satisfechos respecto a la media aritmética y teniendo en cuenta las puntuaciones establecidas por la encuesta; sin embargo, se puede observar la ponderación más baja en las variables de condiciones básicas de infraestructura y sentido de pertenencia a la institución y al grupo de clase, es decir están poco satisfechos. En lo que respecta a las variables más altas, se encuentran: las condiciones de seguridad y reconocimiento al éxito personal, es decir muy satisfechos. De acuerdo con Álvarez Botello et al. (2015) las valoraciones que mide la calidad de educativa, están dadas por los estudiantes y la unidad de aprendizaje (herramientas, equipamientos y espacios) que otorga la institución. Si las instalaciones no cuentan con estos requisitos, se verían afectadas las rutas de aprendizaje de los estudiantes debido a que no se les garantiza las condiciones mínimas para la apropiación del conocimiento.

Reforzando lo anterior, Bernal, Martínez, Parra, y Jiménez (2015) sostienen que lograr una confortabilidad a nivel estructural de las instalaciones en las instituciones de educación superior, ayuda al desarrollo social y personal de los estudiantes. Se puede entonces resaltar que una parte de la calidad educativa está dada por las condiciones en las que se encuentran las universidades $\mathrm{y}$ en los espacios $\mathrm{o}$ materiales que se ofrecen, para los procesos de enseñanza y aprendizaje de los estudiantes.

En el mismo enfoque es preciso recomendar que las instituciones universitarias, tengan en cuenta los factores que pueden ayudar a que los estudiantes sientan confortabilidad y satisfacción en su institución, esto para ayudar en la mejora continua y permanente de la calidad educativa, reforzando también la seguridad de los estudiantes, a través del uso de salones 
bien dotados, adecuados servicios sanitarios y planes de riesgos en caso de emergencia. Arciniegas Ortiz y Mejías Acosta (2017) aseveran que la satisfacción de los alumnos en aspectos como la seguridad, está ligada con las instalaciones ofrecidas por las instituciones y también con el equipamiento que estas tienen. Mientras Arancibia Carvajal, Donoso Pérez, Montenegro Iturra y Díaz Farías (2014) resaltan que para lograr óptimos procesos de aprendizaje y enseñanza en las instituciones, se requiere de un estado adecuado de la infraestructura, además de contar con todos los requisitos de salubridad $\mathrm{y}$ seguridad que protegen a los miembros que la conforman.

En lo referente al sentido de pertenecía a la institución y al grupo de clase por la aceptación, se reflejó una ponderación muy baja, con lo que se puede inferir que los estudiantes de la licenciatura se encuentran poco satisfechos. Brea (2014) recomienda que si se está completamente satisfecho con este aspecto en el medio educativo, se fortalecerán los procesos psicológicos de los estudiantes evidenciando mejores posturas hacia la comunidad, maestros e institución. En el ámbito universitario es importante que los estudiantes mantengan sentido de pertenencia hacia la institución ya que es aquí donde obtienen sus conocimientos como profesionales, además de su desarrollo individual y colectivo.

La satisfacción personal es muy importante, debido a que esta de refleja en el rendimiento académico; además, los estudiantes denotan si están o no satisfechos con el programa académico y con la institución de educación superior que escogieron. Asimismo, Urrea, Bernate, Fonseca, y Martínez (2018) afirman que existen determinantes extrínsecos, como las diferencias sociales, el entorno familiar, nivel educativo de los progenitores, entre otros. Con ello, se puede afirmar que es importante para los estudiantes, que tanto las instituciones como los docentes dispongan rutas de apoyo y diversidad de estrategias evaluativas para lograr una satisfacción óptima.

La variable de reconocimiento del éxito personal, realmente sintetiza la satisfacción de cualquier aspecto de la vida, para el caso del ámbito universitario, los estudiantes son el eje fundamental de toda institución educativa, debido a que son ellos los que definen el grado de satisfacción, por lo tanto su visión debe tomarse en cuenta para mostrar los aspectos a mejorar. García-Rangel, García Rangel, y Reyes Angulo (2014) aluden, que los profesores pueden colaborar con el éxito o el fracaso de los estudiantes por medio de sus clases, es decir, que cumplen un rol preponderante en los universitarios, denotando la misión que tiene el docente en las aulas de clase y en la vida académica del estudiante.

Es importante tener en cuenta la variable de auto relación, dado que converge con las variables anteriores, pues contempla lo que el estudiante es capaz de hacer en términos del saber, de las habilidades adquiridas durante su 
carrera, además dela autonomía y la creatividad en los diferentes espacios académicos. Al respecto Salazar Yépez y Cabrera-Vallejo (2016) afirman, que el maestro debe sensibilizarse frente a las situaciones que afectan el proceso de aprendizaje de los estudiantes. Por lo tanto, durante el recorrido que hace el estudiante en la institución, se deberá materializar el conocimiento y los valores que este adquiere mediante su proceso de formación profesional. Asimismo, el maestro entrará en el contexto de sus estudiantes centrándose en sus logros y metas.

\section{Conclusiones}

Como resultante del trabajo investigativo, se logró reconocer que aunque la institución educativa ha centrado esfuerzos y recursos de diferente orden (económicos, humanos, didácticos, entre otros) en la actualidad los estudiantes no alcanzan satisfacción frente al tema de infraestructura dado que aún no se cuenta con los escenarios deportivos suficientes para el pleno ejercicio de las actividades prácticas propias del programa de Licenciatura en Educación Física.

En lo que respecta a la variable del grupo VI: Sentido de pertenencia a la institución, se refleja que los estudiantes están poco satisfechos, esto puede estar relacionado con la baja participación de los mismos en la gestación de políticas y proyectos institucionales, en tanto no ven reflejados los cambios en el contexto universitario en el que siguen su formación profesional.
Por último, la investigación permite concluir que los estudiantes se encuentran satisfechos en la mayoría de las variables analizadas, lo que da cuenta de la ruta seguida por la universidad para el logro de los objetivos de calidad que permitan maximizar el proceso educativo en pro de aportar e impactar positivamente la sociedad Colombiana.

\section{Referencias}

Arciniegas Ortiz, J. A., y Mejías Acosta, A. A. (2017). Percepción de la calidad de los servicios prestados por la Universidad Militar Nueva Granada con base en la escala Servqualing, con análisis factorial y análisis de regresión múltiple. Comuni@cción,8(1),26-36.

Aguilera García, J. L. (2010). La tutoría en la universidad: selección, formación y práctica de los tutores: ajustes para la UCM desde el Espacio Europeo de Educación Superior (Doctoral dissertation, Universidad Complutense de Madrid, Servicio de Publicaciones). https://eprints.ucm.es/10147/

Alcántar Enríquez, V. M., Maldonado-Radillo, S. E., \& Arcos Vega, J. L. (2012). Medición Del Clima Laboral Requerido Para Asegurar La Efectividad Del Sistema De Gestión De Calidad. Revista Internacional Administración \& Finanzas, 5(3), 55-68.

Álvarez Botello, J., Chaparro Salinas, E., y Reyes Pérez, D. (2015). Estudio de la Satisfacción de los Estudiantes con los Servicios Educativos Brindados por Instituciones de Educación Superior del Valle de Toluca. REICE. Revista Iberoamericana Sobre Calidad, Eficacia Y Cambio En Educación, 13(2), 5-26.

Arancibia Carvajal, S., Donoso Pérez, M., Montenegro Iturra, E., y Díaz Farías, A. (2014). Un 
modelo para apoyar la gestión de proyectos de inversión en infraestructura escolar. In the twelfth Latin American and Caribbean conference for engineering and technology (LACCEI 2014). Julio 22-24, 2014, Guayaquil-Ecuador.

Bernal, D., Martínez, M., Parra, A., y Jiménez, J.L. (2015). Investigación Documental Sobre Calidad De La Educación En Instituciones Educativas Del Contexto Iberoamericano. Entramados- Educación y Sociedad, 2(2), 107- 124.

Bernate, J., Fonseca, I., y Betancourt, M. (2019). Impacto de la actividad física y la práctica deportiva en el contexto social de la educación superior. Retos, 37(37), 742-747.

Bianchetti, A. F. (2017). Calidad educativa: concepciones y debate. Revista electrónica de investigación educativa, 19(1), 1-3. Recuperado en 09 de junio de 2020.

Brea, L. M. (2014). Factores determinantes del sentido de pertenencia de los estudiantes de Arquitectura de la Pontificia Universidad Católica Madre y Maestra, Campus Santo Tomás de Aquino (Doctoral dissertation), Universidad de Murcia.

Cadena-Badilla, M., Mejías Acosta, A., Vega-Robles, A., y Vásquez Quiroga, J. (2016). La satisfacción estudiantil universitaria: análisis estratégico a partir del análisis de factores. Industrial Data, 18(1), 9-18.

Camacho, Á. S., \& Delgado Noguera, M. Á. (2002). Educación física y estilos de enseñanza: análisis de la participación del alumnado desde un modelo socio-cultural del conocimiento escolar (Vol. 23). Inde Publicaciones.

Clemenza, C., Ferrer, J., y Pelekais, C. (2005). La calidad como elemento competitivo en las Universidades. Revista de Artes y
Humanidades UNICA,(14), 55-83.

Contreras, K., Caballero, C., Palacio, J., y Pérez, A. M. (2008). Factores asociados al fracaso académico en estudiantes universitarios de Barranquilla (Colombia). Psicología desde el Caribe, (22), 110-135.

Dos Santos, M.. (2016). Calidad y satisfacción: El caso de la Universidad de Jaén.. Revista de la educación superior, 45(178), 79-95. https://dx.doi.org/10.1016/j.resu.2016.02. 005

Fernández-Abascal, E. G., y Jiménez Sánchez, M. P. (2010). Psicología de la emoción. Editorial Universitaria Ramón Areces.

García-Rangel, E. G., García Rangel, A. K., y Reyes Angulo, J. A. (2014). Relación maestro alumno y sus implicaciones en el aprendizaje. Ra Ximhai, 10(5), 279-290.

Gento-Palacios, S., y Vivas-García, M. (2003). El SEUE: un instrumento para conocer la satisfacción de los estudiantes universitarios con su educación. Acción pedagógica, 12(2), 16-27.

Guzmán, J. C. (2011). La calidad de la enseñanza en educación superior ¿Qué es una buena enseñanza en este nivel educativo? Perfiles educativos, 33, 129-141..

Hernández-Sampieri, R., Fernández-Collado, C., y Baptista Lucio, P. (2010). Metodología de la investigación (5ta. edición). México DF: McGraw-Hill Educación.

Mejías, A., y Martínez, D. (2009). Desarrollo de un instrumento para medir la satisfacción estudiantil en educación superior. Docencia Universitaria, 10(2), 29-47.

Orozco Cruz, J. C., Olaya Toro, A., y Villate Duarte, V. (2009). ¿Calidad de la educación o educación de calidad? Una preocupación más allá del mercado. Revista Iberoamericana de educación, (51), 161-181. 
Salazar Yépez, W., y Cabrera-Vallejo, M. (2016). Diagnóstico de la calidad de servicio, en la atención al cliente, en la Universidad Nacional de Chimborazo-Ecuador. Industrial Data, 19(2) ,13-20.

Surdez, E. G., Sandoval, M. C., y Lamoyi, C. L. (2018). Satisfacción estudiantil en la valoración de la calidad educativa universitaria. Educación y Educadores, 21(1), 9-26. Doi: https://doi.org/10.5294/edu.2018.21.1.1

Urrea, P., Bernate, J., Fonseca, I., y Martínez, A. (2018). Impacto social de los graduados de la Licenciatura en Educación Física, Recreación y Deporte UNIMINUTO. Educación Física Y Ciencia, 20(2), e050. https://doi.org/10.24215/23142561e 050

Vega Cantor, R. (2014). Educational quality a neoliberal notion, own of pedagogical Darwinism. Revista Integra Educativa, 7(2), 113-125.

Villarruel Fuentes, M. (2010). Calidad en la educación superior: Un análisis reflexivo sobre la gestión de sus procesos en los Centros Educativos de América. Revista Iberoamericana sobre Calidad, Eficacia y Cambio en Educación, 8(5), 110-118. 